\title{
Low rates of acceptance of BRCA1 and BRCA2 test results among African American women at increased risk for hereditary breast-ovarian cancer
}

Chanita Hughes Halbert, $P h D^{1}$, Lisa Kessler, $M S^{2}$, Jill E. Stopfer, $M S^{3}$, Susan Domchek, $M D^{4}$, and E. Paul Wileyto, PhD ${ }^{2}$

\begin{abstract}
Purpose: This study evaluated rates of BRCA1 and BRCA2 (BRCA1/2) test result acceptance among African American women and identified determinants of test result acceptance. Methods: Acceptance of $B R C A 1 / 2$ test results was evaluated among 157 African American women at high and moderate risk for having a BRCA1/2 mutation who were offered genetic testing as part of a clinical genetic counseling research program. Results: Twenty-two percent of women received BRCA1/2 test results. Test result acceptance differed between women with $\geq 10 \%$ prior probability of having a $B R C A 1 / 2$ mutation (34\%) and those who had a $5 \%$ prior probability ( $8 \%$ ). Among women with $\geq 10 \%$ prior probability, test result acceptors were most likely to be married $(\mathrm{OR}=5.29,95 \% \mathrm{Cl}=$ $1.82,15.38, P=0.002)$ and be less certain about their risk of developing cancer $(\mathrm{OR}=3.18,95 \% \mathrm{Cl}=1.04$, 9.80, $P=0.04)$. Conclusion: These results demonstrate that acceptance of $B R C A 1 / 2$ test results may be limited among African American women. Being married and having less certainty about one's cancer risk may motivate acceptance of $B R C A 1 / 2$ test results among African American women. It may be important to emphasize the possibility that $B R C A 1 / 2$ test results may not clarify cancer risks during pre-test counseling with African American women to ensure informed decision-making about testing. Genet Med 2006:8(9):576-582.
\end{abstract}

Key Words: African American, BRCA1 and BRCA2, test result, acceptance

Recently, epidemiological studies have shown that the prevalence of BRCA1 and BRCA2 (BRCA1/2) mutations range from 16-28\% among African American women who have a personal and family history of breast and/or ovarian cancer suggestive of hereditary disease. ${ }^{1-4}$ If found to carry a $B R C A 1 / 2$ mutation, women have an estimated $60-80 \%$ lifetime risk of developing breast cancer and a $10-45 \%$ lifetime risk of developing ovarian cancer. ${ }^{5-7}$ Because of the excess rates of breast cancer mortality among African American women, ${ }^{8,9}$ participation in genetic counseling and testing may be beneficial to women at increased risk for hereditary cancer to increase knowledge about cancer risks and options for risk reduction. Efforts are now being made to enhance access to genetic counseling and testing for BRCA1/2 mutations among African American women at increased risk for hereditary disease. Recent research has shown that as many as $50 \%$ of African American women may participate in genetic counseling for breast cancer susceptibility, ${ }^{10}$ but little is known about rates of

From the ${ }^{I}$ Department of Psychiatry, Abramson Cancer Center, and Leonard Davis Institute of Health Economics, University of Pennsylvania, Philadelphia, Pennsylvania; ${ }^{2}$ Department of Psychiatry, University of Pennsylvania, Philadelphia, Pennsylvania; ${ }^{3}$ Abramson Cancer Center, University of Pennsylvania, Philadelphia, Pennsylvania; ${ }^{4}$ Department of Medicine and Abramson Cancer Center, University of Pennsylvania, Philadelphia, Pennsylvania.

Chanita Hughes Halbert, PhD, University of Pennsylvania, 3535 Market Street, Suite 4100, Philadelphia, PA 19104.

Submitted for publication January 3, 2006.

Accepted for publication June 19, 2006.

DOI: 10.1097/01.gim.0000237719.37908.54 acceptance of $B R C A 1 / 2$ test results or determinants of test result acceptance.

To address this gap in our knowledge, we evaluated rates of $B R C A 1 / 2$ test result acceptance among African American women at increased risk for hereditary breast and ovarian cancer and identified sociodemographic, clinical, and psychological barriers and facilitators to receiving genetic test results. Because prior studies have shown that cancer-specific worry may influence decisions about participating in genetic counseling among African American women ${ }^{11}$ we were interested in exploring the relationship between $B R C A 1 / 2$ test result acceptance and cancer-specific worry. Other reports have shown that many African American women would want to have genetic testing to be reassured about their cancer risk ${ }^{12}$; however, it is possible that women who are uncertain about their risk of developing cancer may be most likely to receive test results to better define their risk of disease. Thus, we were also interested in determining whether certainty about one's risk of developing breast cancer is associated with genetic test acceptance. Since previous research has shown that responses to education about hereditary breast cancer and genetic testing may differ among African American women depending on the extent to which information addresses individual concerns, ${ }^{11}$ a secondary aim of the study was to explore whether two forms of pre-test counseling, culturally tailored versus standard, influence acceptance of $B R C A 1 / 2$ test results among women who participate in pre-test counseling. Information on rates and determinants of $B R C A 1 / 2$ test result acceptance will provide important informa- 
tion on uptake of this service among African American women at increased risk for hereditary breast and ovarian cancer.

\section{MATERIALS AND METHODS}

\section{Study population}

Participants were African American women $(\mathrm{N}=157)$ at increased risk for having a $B R C A 1 / 2$ mutation. To be eligible for participation, women had to self-identify as being African American or Black and have at least a $5-10 \%$ prior probability of having a BRCA1/2 mutation based on their personal and family history of breast and/or ovarian cancer. Prior probability of having a BRCA1/2 mutation was estimated based on the participant's personal and family history of breast and/or ovarian cancer using risk estimation models and empiric data from prior reports. ${ }^{3,13-15}$ The study was approved by the Institutional Review Board at the University of Pennsylvania.

\section{Procedures}

Women were recruited into the study though a referral network that included seven clinical institutions and community oncology resources located in Philadelphia, PA. At the clinical referral sites, brochures and flyers that contained information about the study were given to all African American women by physicians and clinic staff. Study brochures and flyers were given to women by research staff at community oncology resources. Women interested in learning more about genetic counseling completed a referral form that collected information on race, address, birth date, and personal and family history of cancer. Eligibility was determined by the study genetic counselor following referral and eligible women were mailed an invitation letter that described the study purpose and procedures involved in participation. Some women $(\mathrm{N}=27)$ were referred from a separate epidemiological study that was designed to identify genetic risk factors for breast cancer in African American women and had provided a blood sample before enrolling in this study. However, these women did not receive genetic counseling for hereditary breast-ovarian cancer susceptibility and clinical genetic testing for BRCA1/2 mutations was not performed. Further, referral from the epidemiological study was not associated with decisions about enrolling in this study. ${ }^{10}$ Study enrollment included completion of a structured baseline telephone interview that took about 40 minutes to complete. Both study enrollment and the baseline were completed by a trained interviewer at Penn after obtaining verbal consent. Project staff who completed the study enrollment and the baseline telephone interview were African American. The baseline assessed sociodemographics, cancer-specific worry, and risk perception variables. The response rate for the baseline telephone interview and study enrollment was $61 \%$ (Fig. 1). At the end of the baseline, women were invited to participate in genetic counseling; those who agreed to participate in counseling were randomized to culturally tailored or standard genetic counseling. Detailed information on these counseling protocols is provided below under "Genetic Counsel- ing Protocols." Women were recruited into the study from February, 2003 through October, 2005.

\section{Genetic counseling protocols}

Standard Genetic Counseling (SGC): Following provision of written informed consent, women randomized to SGC received pre-test counseling about hereditary breast and ovarian cancer, the inheritance and prevalence of $B R C A 1 / 2$ susceptibility genes, the process of genetic testing for $B R C A 1 / 2$ mutations, and interpretation of genetic test results using a semistructured protocol. Risk of having a BRCA1/2 mutation was also provided to women along with information about cancer risks associated with $B R C A 1 / 2$ mutations and the potential benefits, limitations, and risks of genetic testing. Possible test result outcomes (e.g., positive, negative, or variant of unknown significance) were also reviewed. The SGC session lasted about 90 minutes. Similar protocols have been used to provide pretest counseling in prior studies. ${ }^{16,17}$

Culturally tailored genetic counseling (CTGC): The CTGC protocol provided the same education about hereditary cancer, genetic testing, and risk information as the SGC protocol after written informed consent was obtained. However, consistent with guidelines for providing culturally competent genetic counseling, ${ }^{18,19}$ the CTGC protocol included standardized probes to elicit discussion about cultural factors that have been shown to influence decisions about genetic counseling among African American women in prior reports (e.g., spiritual and religious beliefs, communalism). ${ }^{20,21}$ For example, women were asked what aspects of their spiritual and religious beliefs influence their decision to have genetic testing to facilitate discussion about the role of these factors in decision-making about genetic testing for $B R C A 1 / 2$ mutations. Women were also asked questions such as how their familial experiences with breast and ovarian cancer influenced their decisions to have genetic testing to facilitate discussions about values related to communalism. The CTGC sessions lasted 90-120 minutes. The study genetic counselor (LK) took detailed counseling notes after CTGC and SGC to document the issues discussed during pre-test counseling and these notes were reviewed by the $\mathrm{PI}(\mathrm{CHH})$ to ensure adherence to the counseling protocols. In addition, counseling sessions were randomly audiotaped and reviewed by the PI to ensure adherence to the counseling protocols. The SGC and CTGC sessions were conducted using a semi-structured protocol that included visual aids to standardize the educational content and a written summary of the educational content was provided to women to refer to after the session. Sessions were conducted individuals by a board certified genetic counselor (LK) who was Caucasian.

At the end of culturally tailored or standard genetic counseling, women were given an opportunity to provide a blood sample for genetic testing. Women who were interested in having genetic testing were scheduled for a meeting with a medical oncologist (SD). During this visit, women discussed any new medical issues and were offered a clinical breast examination. Possible test result outcomes, as well as the risks and benefits of genetic testing, were reviewed by the medical oncologist. Spe- 


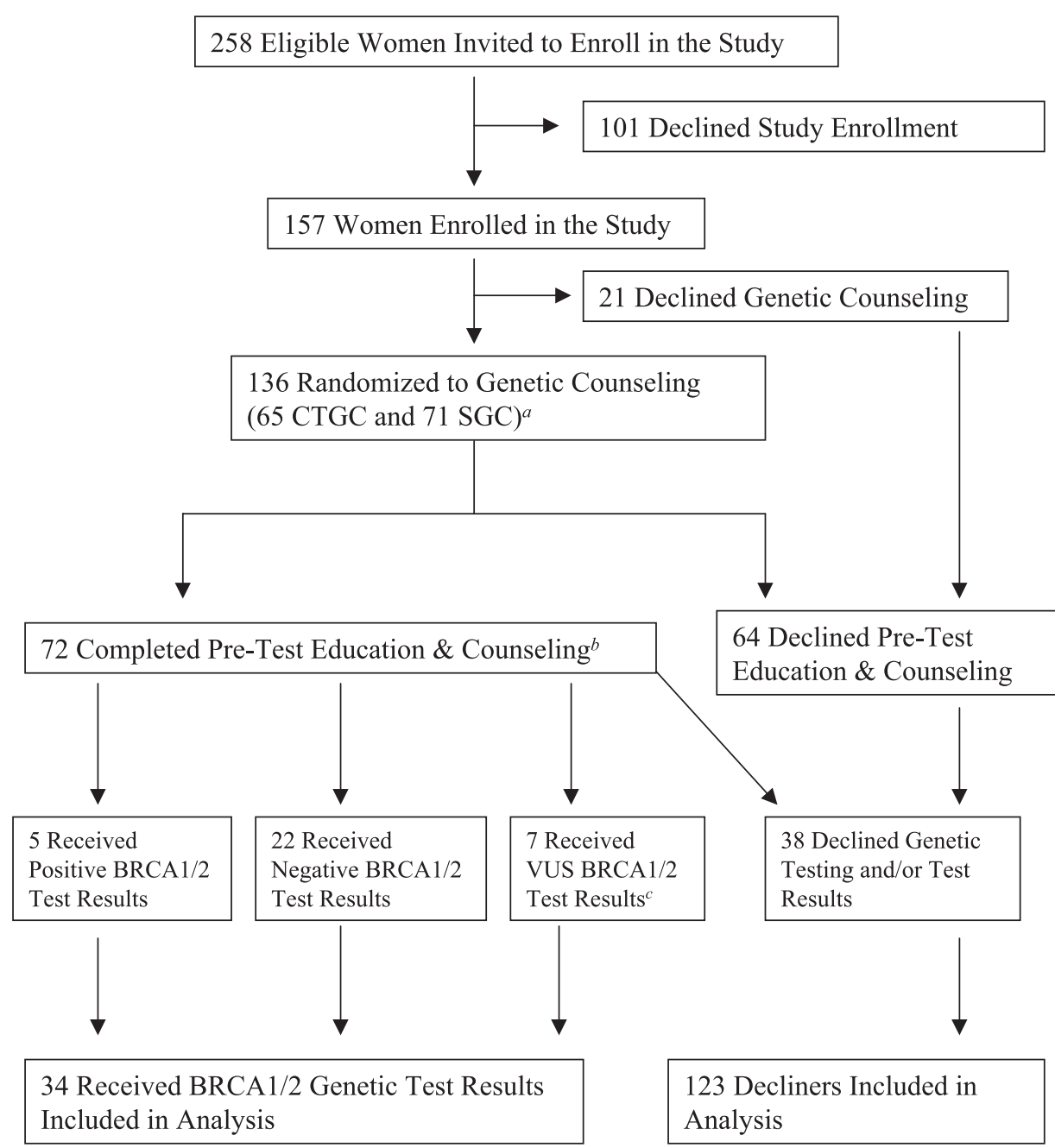

Fig. 1. Flow of Study Procedures. ${ }^{a}$ CTGC, Culturally Tailored Genetic Counseling; SGC, Standard Genetic Counseling. ${ }^{b} 39$ Women who completed pre-test education and counseling provided a blood sample for genetic testing. ${ }^{c}$ VUS, Variant of Unknown Significance.

cific issues that were discussed were the ways that knowledge of BRCA1/2 mutation status might influence medical management (e.g., oophorectomy, enhanced screening) for themselves and their family members, as well as the possibility of variants of unknown significance. Blood samples were obtained from women who were interested in genetic testing following provision of written informed consent at the end of this appointment. When test results became available, women were contacted by telephone by the study genetic counselor to schedule a test results disclosure session. Costs for genetic testing were paid by the participant's insurance company or by institutional funds at the Abramson Cancer Center.

Participants who provided a blood sample were invited to attend an individual test result disclosure and counseling session when their BRCA1/2 test results became available. Following provision of written informed consent, BRCA1/2 test results were disclosed by the genetic counselor and medical oncologist. Women were also provided with information about their risk of developing cancer, individualized guidelines for surveillance and prevention options, and risk of having a BRCA1/2 mutation among family members. Following disclosure of $B R C A 1 / 2$ test results and discussion of guidelines for cancer screening and surveillance, a semistructured culturally tailored protocol was used to facilitate discussion of cultural belief and values that were addressed during the pre-test counseling session among women who were randomized to CTGC. For example, women were asked what aspects of their religious and spiritual beliefs they would use to cope with their BRCA1/2 test results. Women were also asked which family members would they lean on for support following test results disclosure and how would they react if relatives did not want to know their $B R C A 1 / 2$ test results.

Regardless of test result and randomization to CTGC or SGC, all women received a written report that included an interpretation of their BRCA1/2 test result and guidelines for medical management. In addition, all women were contacted by the study genetic counselor approximately two weeks following the test result disclosure session to answer any additional questions and to provide additional referrals, if needed. 


\section{Measures}

\section{Sociodemographics}

Income, marital status, education, and employment status were obtained during the baseline telephone interview. Responses to these items were re-coded into dichotomous variables (e.g., not married vs. married) based on the distribution of responses.

\section{Clinical factors}

Age, personal history of cancer, and family history of disease were obtained by self-report. Women were categorized as being age 50 or younger or older than age 50.

The total number of first-, second-, and third-degree relatives diagnosed with breast and/or ovarian cancer was calculated because it is standard practice to construct a three-generation pedigree for genetic counseling. ${ }^{22}$ Women were categorized as having two or more affected relatives or less than two relatives affected with breast and/or ovarian cancer.

\section{Psychological variables}

Psychological factors were evaluated in terms of certainty about one's risk of developing cancer, perceived risk of having a BRCA1/2 mutation, and cancer-specific worry. Specifically, we used one Likert-style item validated in previous research on genetic counseling for inherited breast cancer risk to evaluate perceived risk of having a BRCA1/2 mutation. ${ }^{11,23}$ Certainty about one's risk of developing cancer was evaluated with a Likert-style item that asked women how certain they were of their chances of getting breast cancer $(1=$ not at all certain, $2=$ a little certain, $3=$ somewhat certain, $4=$ very certain). Similar types of items have been used in prior research to evaluate certainty about one's breast cancer risk. ${ }^{24}$ Responses to these items were re-coded into dichotomous variables based on the distribution of responses (e.g., at risk vs. not at risk and more certain vs. less certain). We used the breast cancer worry scale to evaluate cancer-specific worry. ${ }^{25}$ This questionnaire asked women to indicate how much they thought about their chances of developing breast cancer and how much thoughts about developing breast cancer impacted their mood and ability to perform their daily activities. This scale has been used to measure cancer-specific worry among women seeking genetic counseling for $B R C A 1 / 2$ mutations in previous research ${ }^{26}$ and had good internal consistency in this sample (Cronbach's alpha $=0.76)$.

\section{Acceptance of BRCA1/2 test results}

Women were classified as either BRCA1/2 test result acceptors or decliners. Acceptors included women who participated in genetic counseling, provided a blood sample for testing, and received $B R C A 1 / 2$ test results. As in prior reports, ${ }^{16,27}$ decliners included women who did not receive BRCA1/2 test results within $8-12$ weeks of being notified that results were available, women who declined to participate in genetic counseling, and those who declined to provide a blood sample for testing following pre-test counseling. We compared women who de- clined to participate in genetic counseling to those who declined genetic testing or test results and there were no differences in terms of sociodemographic characteristics (e.g., marital status, $\chi^{2}=0.19, P=0.66$ ), clinical factors (e.g., cancer history, $\chi^{2}=1.28, P=0.26$ ), or psychological variables (e.g., breast cancer certainty, $\chi^{2}=0.13, P=0.72$ ). Costs for genetic testing were paid by institutional funds for women with $\geq 10 \%$ prior probability of having a BRCA1/2 mutation. For women with a $5 \%$ prior probability, these costs were paid by insurance companies.

\section{Data analysis}

We first generated frequencies to characterize participants in terms of sociodemographics, clinical factors, and acceptance of BRCA1/2 test results. Next, we conducted $\chi^{2}$ analysis to evaluate the relationship between randomization to CTGC and SGC and sociodemographics and clinical factors. We then conducted $\chi^{2}$ tests of association to evaluate the relationship between BRCA1/2 test result acceptance and randomization to CTGC and SCG. We then conducted bivariate analyses to evaluate the relationship between $B R C A 1 / 2$ test result acceptance and sociodemographics, clinical factors, and cancer-specific worry using a combination of $\chi^{2}$ tests of association for dichotomous variables and non-parametric analysis of variance for continuous measures. These analyses were stratified by $B R C A 1 / 2$ prior probability because of differences in coverage for genetic testing expenses among women with $\geq 10 \%$ prior probability and those with a $5 \%$ prior probability. We then conducted multivariate logistic regression analysis to identify factors having independent associations with $B R C A 1 / 2$ test result acceptance. Variables that had a bivariate association of $P<0.10$ with test result acceptance were included in the logistic regression model.

\section{RESULTS}

\section{Sample characteristics}

As shown in Table 1, the sample consisted mostly of women who had $\geq 10 \%$ prior probability of having $B R C A 1 / 2$ mutation (53\%). In addition, most women were ages 50 and younger $(61 \%)$, were not married (69\%), had some college education or were college graduates $(69 \%)$, were employed $(62 \%)$, and had an annual household income less than $\$ 35,000$ (52\%). Ninetyseven percent of women had health insurance. There were no differences in sociodemographic characteristics between women who had $\geq 10 \%$ prior probability of having a BRCA1/2 mutation and those who had a $5 \%$ prior probability. Overall, $64 \%$ of women had a personal history of breast and/or ovarian cancer and most women had two or more relatives affected with cancer (59\%). In terms of randomization to genetic counseling, $48 \%$ of women were randomized to CTGC $(\mathrm{N}=65)$ and $52 \%$ were randomized to SGC $(\mathrm{N}=71)$. Women who did not participate in the prior epidemiological study $\left(\chi^{2}=6.95\right.$, $P=0.01)$ and those with a high school education or less $\left(\chi^{2}=\right.$ $6.22, P=0.01)$ were more likely to be randomized to CTGC; however, there were no differences in marital status $\left(\chi^{2}=0.13\right.$, 
Table 1

Sample Characteristics $(\mathrm{N}=157)$

\begin{tabular}{|c|c|c|c|c|c|}
\hline Variable & Level & $\begin{array}{c}\text { Total sample } \\
(\mathrm{N}=157) \mathrm{N} \\
(\%)\end{array}$ & $\begin{array}{l}\geq 10 \% \text { BRCA1/2 prior probability } \\
(\mathrm{N}=83) \mathrm{N}(\%)\end{array}$ & $\begin{array}{c}5 \% \text { BRCA1/2 prior probability } \\
(\mathrm{N}=74) \mathrm{N}(\%)\end{array}$ & $\chi^{2}$ \\
\hline \multirow[t]{2}{*}{ Age } & $\leq 50$ & $95(61 \%)$ & $54(65 \%)$ & $41(55 \%)$ & 1.52 \\
\hline & $>50$ & $62(39 \%)$ & $29(35 \%)$ & $33(45 \%)$ & \\
\hline \multirow[t]{2}{*}{ Marital status } & Not married & $109(69 \%)$ & $54(65 \%)$ & $55(74 \%)$ & 1.58 \\
\hline & Married & $48(31 \%)$ & $29(35 \%)$ & $19(26 \%)$ & \\
\hline \multirow[t]{2}{*}{ Education level } & $\geq$ Some college & $109(69 \%)$ & $58(70 \%)$ & $51(69 \%)$ & 0.02 \\
\hline & $\leq$ High school & $48(31 \%)$ & $25(30 \%)$ & $23(31 \%)$ & \\
\hline \multirow[t]{2}{*}{ Employment status } & Employed & $98(62 \%)$ & $48(58 \%)$ & $50(68 \%)$ & 1.58 \\
\hline & Not employed & $59(38 \%)$ & $35(42 \%)$ & $24(32 \%)$ & \\
\hline \multirow[t]{2}{*}{ Income level } & $<\$ 35,000$ & $82(52 \%)$ & $45(54 \%)$ & $37(51 \%)$ & 0.19 \\
\hline & $>\$ 35,000$ & $74(48 \%)$ & $38(46 \%)$ & $36(49 \%)$ & \\
\hline \multirow[t]{2}{*}{ Insurance status } & Yes & $152(97 \%)$ & $81(98 \%)$ & $71(96 \%)$ & 0.34 \\
\hline & No & $5(3 \%)$ & $2(2 \%)$ & $3(4 \%)$ & \\
\hline
\end{tabular}

$P=0.72)$, income $\left(\chi^{2}=0.01, P=0.93\right)$, employment $\left(\chi^{2}=\right.$ $1.06, P=0.30)$, cancer status $\left(\chi^{2}=0.14, P=0.70\right)$, family history of cancer $\left(\chi^{2}=0.004, P=0.95\right)$, or $B R C A 1 / 2$ prior probability $\left(\chi^{2}=0.96, P=0.33\right)$ between women randomized to CTGC and SGC.

\section{Acceptance of genetic test results}

There were no differences in BRCA1/2 test result acceptance in the total sample of women who were randomized to CTGC and SGC $(\mathrm{N}=136)$ (22 vs. $28 \%, \chi^{2}=0.80, P=0.37$ ) or among women who participated in pre-test counseling. Among participants in pre-test counseling, $47 \%$ were test result acceptors and 53\% declined. Since there were no differences in test result acceptance among women randomized to CTGC or SGC, we evaluated rates of test result acceptance in the total sample of women who enrolled in the study. Among all women $(\mathrm{N}=$ 157), 22\% were test result acceptors and $78 \%$ were decliners; however, test result acceptance was greater among women who had $\geq 10 \%$ prior probability of having a BRCA1/2 mutation (34\%) compared to those who had a $5 \%$ prior probability $(8 \%)$ $\left(\chi^{2}=15.14, P=0.001\right)$. Of the women who received test results, $15 \%$ were mutation carriers, $65 \%$ were $B R C A 1 / 2$ negative, and $21 \%$ had variants of uncertain significance. Since a small number of women with a $5 \%$ prior probability received BRCA1/2 test results $(\mathrm{N}=6)$, we did not complete analyses to identify factors associated with test result acceptance among these women; thus, the analysis presented below is based on women with $\geq 10 \%$ prior probability who enrolled in the study $(\mathrm{N}=83)$.

Of the sociodemographic factors, only marital status was associated significantly with $B R C A 1 / 2$ test result acceptance. Women who were married were significantly more likely to receive BRCA1/2 test results compared to those who were not married $\left(\chi^{2}=9.16, P=0.002\right)$. In addition, cancer-specific worry was greater among women who received $B R C A 1 / 2$ test results compared to decliners (Kruskal-Wallis $\chi^{2}=2.87, P=0.09$ ). However, women who were less certain about their risk of developing cancer (42\%) were more likely to receive BRCA1/2 test results compared to women who were more certain about their risks $(22 \%)\left(\chi^{2}=\right.$ $3.51, P=0.06)$. No other sociodemographic, clinical factors, or psychological variables were associated significantly with BRCA1/2 test result acceptance.

\section{Predictors of test result acceptance}

In the multivariate logistic regression model of acceptance of $B R C A 1 / 2$ test results, only marital status and certainty about breast cancer risk had significant independent associations with test result acceptance. As shown in Table 2, women who were married were about five times more likely than unmarried women to receive $B R C A 1 / 2$ test results $(\mathrm{OR}=5.29,95 \% \mathrm{CI}=1.82,15.38$, $P=0.002)$. In addition, women who were less certain about their cancer risk were about three times more likely to receive $B R C A 1 / 2$

\section{Table 2}

Multivariate logistic regression model of $B R C A 1$ and $B R C A 2$ tes result acceptance ${ }^{a}$

\begin{tabular}{lccc}
\hline Variable & Estimate & SE & OR $(95 \%$ CI $)$ \\
\hline $\begin{array}{l}\text { Marital status, married/ } \\
\text { not married }\end{array}$ & 1.67 & 0.54 & $5.29(1.82,15.38)^{b}$ \\
$\begin{array}{l}\text { Risk certainty, less certain/ } \\
\text { more certain }\end{array}$ & 1.16 & 0.57 & $3.18(1.04,9.80)^{c}$ \\
$\begin{array}{l}\text { Breast cancer worries } \\
{ }^{d}\end{array}$ & 0.12 & 0.10 & $1.35(0.83,2.20)$
\end{tabular}

${ }^{a}$ Only includes women with $\geq 10 \% B R C A 1 / 2$ prior probability; $\mathrm{N}=81$ because of missing data.

${ }^{b} P=002$.

${ }^{c} P=0.04$.

${ }^{d}$ Odds ratio reflects the increase in odds associated with 1 standard deviation increase in the continuous measure of breast cancer worries. 
test results compared to women who were more certain $(\mathrm{OR}=$ $3.18,95 \% \mathrm{CI}=1.04,9.80, P=0.04)$. We re-ran the model controlling for education and participation in the prior epidemiological study and the results were unchanged (marital status, OR = $5.84,95 \% \mathrm{CI}=1.92,17.77, P=0.002$; certainty, $\mathrm{OR}=3.39,95 \%$ $\mathrm{CI}=1.06,10.82, P=0.04)$.

\section{DISCUSSION}

Prior reports have evaluated participation in genetic counseling among African American women; ${ }^{10,20,26}$ however, to our knowledge, this study is the first to document rates of actual $B R C A 1 / 2$ test result acceptance among African American women at increased risk for hereditary breast and ovarian cancer. Overall, $22 \%$ of women received BRCA1/2 test results; once women underwent pre-test counseling, $47 \%$ of women received $B R C A 1 / 2$ test results. These findings suggest that acceptance of BRCA1/2 test results may be limited among African American women at increased risk for hereditary cancer, especially in comparison to acceptance rates reported for other populations. ${ }^{16,27}$ Importantly, however, acceptance rates did not differ between women who received culturally tailored and standard genetic counseling. Cultural beliefs and values are increasingly being recognized as important factors in genetic counseling 18,19,28 and our recent study found that African American women who received culturally tailored genetic counseling were more satisfied with some aspects of counseling compared to those who received standard genetic counseling. ${ }^{29}$ However, the effect of genetic counseling on BRCA1/2 test result acceptance was based on a limited number of women who completed pre-test counseling; thus, this finding should be interpreted with caution.

The results of this study provide some insight into factors that are likely to motivate acceptance of BRCA1/2 test results among African American women. We found that women who were less certain about their risk of developing breast cancer were about three times more likely to receive $B R C A 1 / 2$ test results compared to women who were more certain about their risks. Provision of risk information is a key component of genetic counseling for BRCA1/2 mutations ${ }^{30,31}$ and previous research has shown that obtaining information about cancer risks is an important motivation for genetic testing among African American women. ${ }^{12}$ However, recent research has shown that many African American women may have BRCA1/2 variants of unknown significance ${ }^{4}$; thus, genetic testing may not clarify cancer risks for these women. This underscores the importance of preparing African American women for this possible outcome during pre-test counseling and ensuring that women understand the clinical implications of genetic test results as part of test results disclosure.

We also found that women who were married were most likely to receive $B R C A 1 / 2$ test results whereas cancer-specific worry did not have a significant effect on BRCA1/2 test result acceptance. Previous research has demonstrated that guilt about passing a BRCA1/2 mutation to relatives may be a barrier to participation in genetic counseling among African American women. ${ }^{20}$ However, women are likely to discuss genetic testing with their partner before making a decision about testing. ${ }^{32}$ It is possible that married women may have been encouraged to have testing by their spouses and/or partners (Hughes, unpublished data, 1997). Spouses are an important resource for emotional support following breast cancer diagnosis among African American women ${ }^{33}$; the availability of spousal and/or partner support following test results disclosure may have also motivated women to receive BRCA1/2 test results. Thus, while cancer-specific worry may not be a barrier to $B R C A 1 / 2$ test result acceptance among African American women, lack of encouragement or support from spouses and/or partners may decrease acceptance of genetic test results.

In considering the results of this study, some limitations should be noted. First, rates of genetic test acceptance were based on $61 \%$ of eligible women who enrolled in the study. The challenges associated with recruiting African American women to participate in cancer research are well-documented ${ }^{34-37}$; however, the enrollment rates for the present study are similar to the rates reported in studies that evaluated genetic testing decisions in Caucasian samples. ${ }^{27,38}$ An additional limitation is that we had limited statistical power to detect small differences in test result acceptance rates between women randomized to CTGC and SGC and the model predicting BRCA1/2 test result acceptance was based only on women with $\geq 10 \%$ prior probability of having a mutation. However, to our knowledge, our report includes the largest sample of African American women at increased risk for hereditary breast cancer to be enrolled in a prospective randomized trial and we had $80 \%$ power to detect moderate effects in the total sample of women randomized to CTGC and SGC and in the subset of women included in the model predicting test result acceptance. Nonetheless, additional research is needed to evaluate acceptance of $B R C A 1 / 2$ test results in larger samples of African American women. Since decliners included women who declined genetic counseling as well as those who declined testing or results, additional research may be needed to evaluate testing decisions based on more uniform groups of women who choose not to participate in genetic counseling, decline genetic testing, or elect to not receive results. However, women who declined genetic counseling did not differ from those who declined testing and/or results in terms of sociodemographic characteristics, clinical factors, or psychological variables. Previous research has shown that racial concordance with health care providers may be important for effective communication; ${ }^{39}$ the lack of racial concordance between participants and the genetic counselor may explain the low rates of genetic test acceptance observed in this study. However, the majority of African American women were extremely satisfied with genetic counseling even though they were not racially concordant with the counselor. ${ }^{29}$ Thus, we do not believe that racial discordance between the counselor and participants was a factor in decisions about genetic testing. However, this is an important area for future research.

Despite these potential limitations, the results of this study demonstrate that acceptance of BRCA1/2 test results may be limited among African American women. Since lack of spousal/partner support may be a barrier to acceptance of $B R C A 1 / 2$ test results among African American women, it may be useful to identify 
other resources for support as women considering testing. Previous research has shown that individuals who have more cohesive relationships with family members are most likely to receive BRCA1/2 test results. ${ }^{40}$ Thus, other family members might be able to provide support to women who are not married as these individuals consider genetic testing for $B R C A 1 / 2$ mutations. Since African American women may be likely to receive $B R C A 1 / 2$ test results to clarify their risks of developing cancer, our results also underscore the importance of discussing possible testing outcomes and the likelihood that $B R C A 1 / 2$ test results may not clarify cancer risks as part of pre-test counseling with African American women to ensure that women make informed decisions about testing. Additional research is needed to understand the effects of $B R C A 1 / 2$ test results, especially uncertain risk information, on psychological functioning and cancer screening behaviors among African American women.

\section{ACKNOWLEDGMENTS}

This research was supported by Department of Defense grant \#DAMD17-00-1-0262. Costs for genetic testing for BRCA1 and BRCA2 mutations were covered in part by institutional funds at the Abramson Cancer Center. We would like to acknowledge Aliya Collier, BA, Kiyona Brewster, MA, ChaChira Smith, and Benita Weathers, MPH, for assistance with data collection and management. We are very appreciative to all of the women who participated in this research.

\section{References}

1. Panguluri RC, Brody LC, Modali R, Utley K, et al. BRCA1 mutations in African Americans. Hum Genet 1999;105:28-31.

2. Gao Q, Tomlinson G, Das S, Cummings S, et al. Prevalence of BRCA1 and BRCA2 mutations among clinic-based African American families with breast cancer. Hum Genet 2000;107:186-191.

3. Frank TS, Deffenbaugh AM, Reid JE, Hulick M, et al. Clinical characteristics of individuals with germline mutations in BRCA1 and BRCA2: analysis of 10,000 individuals. J Clin Oncol 2002;20:1480-1490.

4. Nanda R, Schumm LP, Cummings S, Fackenthal JD, et al. Genetic testing in an ethnically diverse cohort of high-risk women: a comparative analysis of BRCA1 and BRCA2 mutations in American families of European and African ancestry. JAMA 2005;294:1925-1933.

5. Easton DF, Ford D, Bishop DT. Breast and ovarian cancer incidence in BRCA1 mutation carriers. Breast Cancer Linkage Consortium. Am J Hum Genet 1995;56:265-271.

6. Ford D, Easton DF, Stratton M, Narod S, et al. Genetic heterogeneity and penetrance analysis of the BRCA1 and BRCA2 genes in breast cancer families. The Breast Cancer Linkage Consortium. Am J Hum Genet 1998;62:676-689.

7. Antoniou A, Pharoah PD, Narod S, Risch HA, et al. Average risks of breast and ovarian cancer associated with BRCA1 or BRCA2 mutations detected in case Series unselected for family history: a combined analysis of 22 studies. Am J Hum Genet 2003;72:1117-1130

8. Field TS, Buist DS, Doubeni C, Enger S, et al. Disparities and survival among breast cancer patients. J Natl Cancer Inst Monogr 2005;88-95.

9. Jemal A, Murray T, Ward E, Samuels A, et al. Cancer statistics, 2005. CA Cancer J Clin 2005;55:10-30.

10. Halbert CH, Brewster K, Collier A, Smith C, et al. Recruiting African American women to participate in hereditary breast cancer research. J Clin Oncol 2005;23: 7967-7973.

11. Lerman C, Hughes C, Benkendorf JL, Biesecker B, et al. Racial differences in testing motivation and psychological distress following pre-test education for BRCAl gene testing. Cancer Epidemiol Biomarkers Prev 1999;8:361-367.
12. Kessler L, Collier A, Brewster K, Smith C, et al. Attitudes about genetic testing and genetic testing intentions in African American women at increased risk for hereditary breast cancer. Genet Med 2005;7:230-238.

13. Couch FJ, DeShano ML, Blackwood MA, Calzone K, et al. BRCAl mutations in women attending clinics that evaluate the risk of breast cancer. N Engl J Med 1997; 336:1409-1415.

14. Parmigiani G, Berry D, Aguilar O. Determining carrier probabilities for breast cancer-susceptibility genes BRCA1 and BRCA2. Am J Hum Genet 1998;62:145-158.

15. Domchek SM, Eisen A, Calzone K, Stopfer J, et al. Application of breast cancer risk prediction models in clinical practice. J Clin Oncol 2003;21:593-601.

16. Schwartz MD, Hughes C, Roth J, Main D, et al. Spiritual faith and genetic testing decisions among high-risk breast cancer probands. Cancer Epidemiol Biomarkers Prev 2000;9:381-385.

17. Halbert CH, Schwartz MD, Wenzel L, Narod S, et al. Predictors of cognitive appraisals following genetic testing for BRCA1 and BRCA2 mutations. J Behav Med 2004; 27:373-392.

18. Weil J. Psychosocial genetic counseling. New York: Oxford University Press, 2000.

19. Weil J. Multicultural education and genetic counseling. Clin Genet 2001;59:143149 .

20. Thompson HS, Valdimarsdottir HB, Duteau-Buck C, Guevarra J, et al. Psychosocia predictors of BRCA counseling and testing decisions among urban African American women. Cancer Epidemiol Biomarkers Prev 2002;11:1579-1585.

21. Hughes C, Fasaye GA, LaSalle VH, Finch C. Sociocultural influences on participation in genetic risk assessment and testing among African American women. Patien Educ Couns 2003;51:107-114.

22. Baker DL, Schuette JL, Uhlmann WR. A guide to genetic counseling. New York: Wiley-Liss, 1998

23. Lerman C, Biesecker B, Benkendorf JL, Kerner J, et al. Controlled trial of pre-test education approaches to enhance informed decision-making for BRCA1 gene testing. J Natl Cancer Inst 1997;89:148-157.

24. Rowe JL, Montgomery GH, Duberstein PR, Bovbjerg DH. Health locus of control and perceived risk of breast cancer in healthy women. Behav Med 2005;31:33-40.

25. Lerman C, Trock B, Rimer BK, Jepson C, et al. Psychological side effects of breast cancer screening. Health Psychol 1991;10:259-267.

26. Armstrong K, Micco E, Carney A, Stopfer J, et al. Racial differences in the use of BRCA1/2 testing among women with a family history of breast or ovarian cancer. JAMA 2005;293:1729-1736.

27. Lerman C, Narod S, Schulman K, Hughes C, et al. BRCA1 testing in families with hereditary breast-ovarian cancer. A prospective study of patient decision making and outcomes. JAMA 1996;275:1885-1892.

28. Fisher NL. Cultural and ethnic diversity: a guide for genetics professionals. Baltimore: Johns Hopkins University Press, 1996.

29. Charles S, Kessler L, Stopfer JE, Domchek S, et al. Satisfaction with genetic counseling for BRCA1 and BRCA2 mutations among African American women. Patient Educ Couns In press.

30. Peters JA, Stopfer JE. Role of the genetic counselor in familial cancer. Oncology 1996;10:159-166.

31. Stopfer JE. Genetic counseling and clinical cancer genetics services. Semin Surg Oncol 2000;18:347-357.

32. Manne S, Audrain J, Schwartz M, Main D, et al. Associations between relationship support and psychological reactions of participants and partners to BRCAl and BRCA2 testing in a clinic-based sample. Ann Behav Med 2004;28:211-225.

33. Morgan PD, Fogel J, Rose L, Barnett K, et al. African American couples merging strengths to successfully cope with breast cancer. Oncol Nurs Forum 2005;32:979-987.

34. Ashing-Giwa K. The recruitment of breast cancer survivors into cancer control studies: a focus on African American women. J Natl Med Assoc 1999;91:255-260.

35. Ashing-Giwa KT, Padilla GV, Tejero JS, Kim J. Breast cancer survivorship in a multiethnic sample: challenges in recruitment and measurement. Cancer 2004;101: $450-465$.

36. Moorman PG, Skinner CS, Evans JP, Newman B, et al. Racial differences in enrolment in a cancer genetics registry. Cancer Epidemiol Biomarkers Prev 2004;13:1349-1354.

37. Murthy VH, Krumholz HM, Gross CP. Participation in cancer clinical trials: race-, sex-, and age-based disparities. JAMA 2004;291:2720-2726.

38. Lerman C, Hughes C, Trock BJ, Myers RE, et al. Genetic testing in families with hereditary nonpolyposis colon cancer. JAMA 1999;281:1618-1622.

39. Cooper LA, Roter DL, Johnson RL, Ford DE, et al. Patient-centered communication, ratings of care, and concordance of patient and physician race. Ann Intern Med 2003;139:907-915.

40. Biesecker BB, Ishibe N, Hadley DW, et al. Psychosocial factors predicting BRCAl/ BRCA2 testing decisions in members of hereditary breast and ovarian cancer families. Am J Med Genet 2000;93:257-263. 